\title{
Vibrational Phase Contrast Microscopy by Use of Coherent Anti-Stokes Raman Scattering
}

\author{
M. Jurna, ${ }^{1}$ J. P. Korterik, ${ }^{1}$ C. Otto, ${ }^{2}$ J. L. Herek, ${ }^{1}$ and H. L. Offerhaus ${ }^{1, *}$ \\ ${ }^{1}$ Optical Sciences group, MESA+ Institute for Nanotechnology, Faculty of Science and Technology (TNW), \\ University of Twente, The Netherlands \\ ${ }^{2}$ BioPhysical Engineering group, MESA+ Institute for Nanotechnology, Faculty of Science and Technology (TNW), \\ University of Twente, The Netherlands
}

(Received 5 March 2009; published 23 July 2009)

\begin{abstract}
In biological samples the resonant coherent anti-Stokes Raman scattering signal of less abundant constituents can be overwhelmed by the nonresonant background, preventing detection of those molecules. We demonstrate a method to obtain the phase of the oscillators in the focal volume that allows discrimination of those hidden molecules. The phase is measured with respect to the local excitation fields using a cascaded phase-preserving chain. It is measured point-by-point and takes into account refractive index changes in the sample, phase curvature over the field-of-view, and interferometric instabilities. The detection of the phase of the vibrational motion can be regarded as a vibrational extension of the linear (refractive index) phase contrast microscopy introduced by Zernike around 1933.
\end{abstract}

DOI: 10.1103/PhysRevLett.103.043905

PACS numbers: 42.65.Dr, 42.87.Bg, 78.47.nj, 87.64.mh

Over the last decade CARS (coherent anti-Stokes Raman scattering) microscopy has developed into a powerful imaging tool for studying biological questions in a noninvasive way $[1,2]$. CARS probes the vibrational modes of molecules, as in Raman imaging, to visualize a specific type of molecule within the sample. The coincidence between the difference frequency of the pump and Stokes input wavelengths with a vibrational mode resonantly enhances the CARS (anti-Stokes) output signal [3]. The CARS signal is also created nonresonantly. Depending on the ratio of resonant to nonresonant molecules in the focal volume, this nonresonant signal can overwhelm a small resonant signal. Samples that contain a lot of water, such as cells, give rise to a significant nonresonant signal, observed as a background over the full image. The intensity differences in such images are not based on chemical selectivity alone, but contain interferences between the resonant and nonresonant signal. Spontaneous Raman imaging lacks a coherent addition of the resonant signal, yielding an acquisition speed that is applicable to slow biological processes. The intensity of the CARS signal can be written as

$$
I_{\mathrm{CARS}}(\omega) \propto\left|\chi_{R}^{(3)}(\omega)+\chi_{\mathrm{NR}}^{(3)}\right|^{2},
$$

where the connection between Raman and CARS is given by

$$
I_{\text {Raman }}(\omega) \propto \operatorname{Im}\left[\chi_{R}^{(3)}(\omega)\right] .
$$

One commonly used method to remove the background is epi-CARS [4], where the CARS signal is detected in the backward direction. This method does not offer discrimination between resonant and nonresonant CARS signal, but between large and small objects in the focal volume, based on phase matching. Small resonant objects give rise to the CARS signal in both the forward and backward direction, whereas the surrounding bulk medium (e.g., nonresonant water) causes the CARS signal in only the forward direction. Polarization CARS [5-7] truly removes the nonresonant signal, but often also rejects a large part of the resonant signal. Time-resolved CARS [8] rejects the nonresonant component by delaying the probe beam to exploit the differences in decay. Stimulated Raman scattering is inherently background free. It requires the detection of a small modulation on a large background but has been demonstrated successfully for imaging $[9,10]$. Dual pump CARS [11] measures the nonresonant background separately, requiring an extra laser input, long integration times, and high stability. Frequency modulation (fm) CARS [12] uses the mixing between the resonant and nonresonant signal to detect the amplitude modulation. It requires less integration time and can reject the nonresonant background, but requires a constant nonresonant background for faithful reproduction of the resonant amplitude. Interferometric CARS [13] achieves rejection of the nonresonant CARS signal, without rejection of resonant signal by direct detection of the amplitude and phase of the CARS signal. The CARS signal is mixed with a stable external reference signal to avoid misinterpretation due to inhomogeneous distribution of the nonresonant background.

In this Letter we employ a cascaded phase-preserving chain to obtain the necessary stable reference signal for interferometric CARS detection without the need for extra lasers. We demonstrate detection of the phase of the CARS signal and detection of the phase of the excitation fields. Subtraction of those two yields the phase of the motion of the molecules with respect to the excitation fields, taking into account the changes in refractive index in the sample, 
the curvature of the field of view, and any other phase disturbances in the sample. This clean phase provides a clean rejection of the nonresonant background and reveals the hidden detail to an arbitrary precision, limited only by imaging speed. This is the first demonstration of the detection of the phase with respect to the excitation fields and the only technique that offers shot noise limited performance and precision that is limited by integration time only. Phase contrast microscopy as introduced by Zernike [14] provided a new level of detail in samples that offered insufficient contrast in the transmission amplitude. Now, some 75 years later, the vibrational phase can be used to reject the nonresonant amplitude and improve the vibrational contrast.

Interferometric detection mixes a reference field, the socalled local oscillator (LO) field, with the generated CARS field. This LO must be phase- and wavelength-locked to the generated CARS signal. It has been shown previously that the LO can be created in bulk media $[13,15]$, using a cascaded phase-preserving chain [16] or by using a polarization CARS scheme [17]. None of these schemes correct for the refractive index changes in the sample or amplitude mixing. In the cascaded phase-preserving chain an optical parametric oscillator (OPO) is synchronously pumped by the second harmonic of a laser source. The CARS signal is generated with the fundamental of the laser source and the idler from the OPO. The signal from the OPO is phase- and wavelength-locked to the generated CARS signal and can therefore be used as the LO [see Fig. 1(a)].

The total intensity on the detector can be written as

$$
I_{\text {detector }}=\left|E_{\mathrm{LO}}\right|^{2}+\left|E_{\mathrm{AS}}\right|^{2}+2 E_{\mathrm{LO}} E_{\mathrm{Ex}} P,
$$

where the CARS field $E_{\mathrm{AS}} \propto E_{\mathrm{Ex}}\left[\chi_{R}^{(3)}+\chi_{\mathrm{NR}}^{(3)}\right]$, the excitation field $E_{\mathrm{Ex}}=E_{\text {Pump }}^{2} E_{\mathrm{Stokes}}$, and $P$ can be expressed as

$$
P=\left\{\left[\chi_{\mathrm{NR}}^{(3)}+\operatorname{Re}\left(\chi_{R}^{(3)}\right)\right] \cos \phi_{\chi}+\left[\operatorname{Im}\left(\chi_{R}^{(3)}\right)\right] \sin \phi_{\chi}\right\},
$$

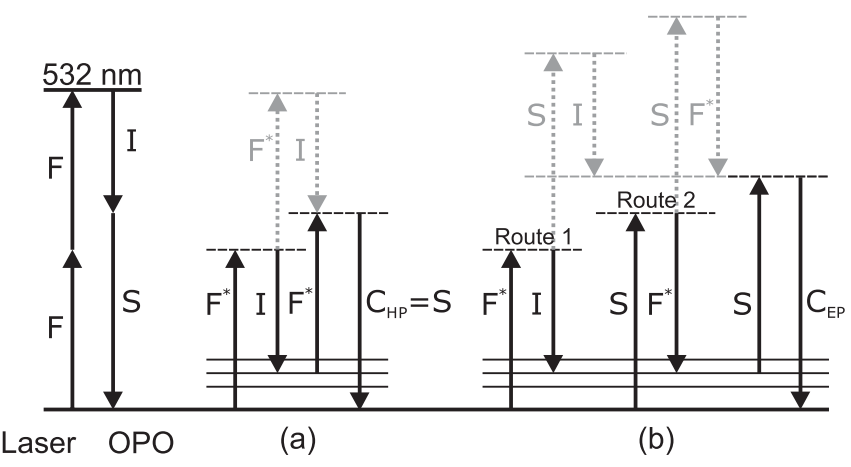

FIG. 1. Schematic of cascaded phase-preserving chain between laser, OPO, and CARS processes. (a) shows the oscillator phase detection scheme, and (b) shows the local excitation phase detection scheme. $F=$ fundamental laser $(1064 \mathrm{~nm}), F^{*}=$ modulated fundamental laser, $I=$ idler OPO, $S=$ signal OPO, and $C=$ CARS signal. In black and gray are, respectively, the resonant and nonresonant CARS processes. where $\phi_{\chi}$ (vibrational phase response) is the phase difference between the total CARS field and the excitation field. This is the phase difference that distinguishes between the real (nonresonant) and the imaginary (resonant, Raman) part [18]. However, this is not directly the phase measured in the focal volume; even for copropagating fields this phase is disturbed by refractive index changes in the sample, curvature of the field of view, and interferometric instabilities. Detection of the local excitation phase and using this "base" phase to correct the interferometric detected phase give the accurate phase of the oscillators in the focal volume, further referred to as a "vibrational phase." For our cascaded chain, the phase difference $\left(\Delta_{1}\right)$ between the local oscillator (the OPO-signal beam) and the CARS signal is given by

$$
\Delta_{1}=\phi_{S}-\left(2 \phi_{F^{*}}-\phi_{I}+\phi_{\chi}\right)=2 \phi_{F}-2 \phi_{F^{*}}-\phi_{\chi}
$$

where $F^{*}$ is a frequency shifted version of $F$. Figure 1(b) shows the detection of the local excitation phase, where there are two separate routes to the same vibrational level (or continuum level) creating an interference that can be probed by the OPO signal and detected at a wavelength given by $2 S-F^{*}$. The interference arises from the phase difference between the two pathways $\left(\Delta_{2}\right)$ :

$$
\Delta_{2}=\left(\phi_{S}-\phi_{F^{*}}\right)-\left(\phi_{F^{*}}-\phi_{I}\right)=2 \phi_{F}-2 \phi_{F^{*}} \text {. }
$$

The difference between the $\Delta_{2}$ and $\Delta_{1}$ is now purely due to the interaction with the vibrational phase response $\phi_{\chi}$. Phase disturbances will occur within the acquisition time of one frame and over the full field of view. Detection on a point-by-point basis over the sample overcomes these interferometric instabilities.

The key elements of the cascaded phase-preserving chain (see Fig. 2) are the laser source, a Nd:YAG laser (Coherent Paladin, $1064 \mathrm{~nm}$ and $532 \mathrm{~nm}$ ), and a synchronously pumped OPO (APE Levante Emerald). This combination provides a well-controlled and stable LO [19]. The input powers of the fundamental and idler are several tens of $\mathrm{mW}$; the power of the LO is only a few $\mathrm{nW}$. To obtain heterodyne interferometric detection the CARS frequency is frequency shifted by an acousto-optical modulator (AOM 1) in the fundamental branch. By detecting the laser repetition rate and using a voltage controlled oscil-

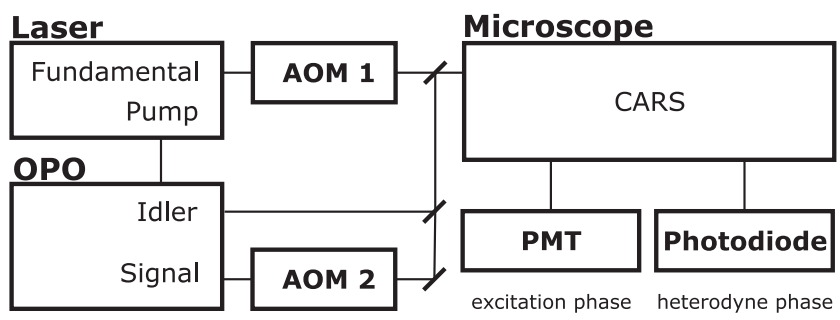

FIG. 2. Schematic of the setup, where AOM 1 is phase modulated and AOM 2 is amplitude modulated. 
lator, an external frequency of $50 \mathrm{kHz}$ is added to the detected laser repetition rate and applied to the acoustooptical modulator. This $50 \mathrm{kHz}$ shift is translated to a $100 \mathrm{kHz}$ shift at the CARS wavelength (two photons in the CARS process). The CARS signal is detected on a photodiode (shot noise limited [16]) and fed to a lock-in amplifier (Stanford Research SR830) set to detect at $100 \mathrm{kHz}$. For detection of the local excitation phase, the amplitude of the signal of the OPO is now changed from a few $\mathrm{nW}$ to the same level as the fundamental beam to balance the contribution of both pathways. Switching between the two power levels is done by a second acoustooptical modulator (AOM 2) in the OPO-signal branch. This acousto-optical modulator is also used in first order, but driven at the laser repetition rate to prevent frequency modulation. The amplitude modulation $(1 \mathrm{kHz})$ of $\mathrm{AOM}$ 2 is set to a multiple of the galvano scanners in the microscope. Scanning at $1 \mathrm{~ms} /$ pixel results in alternating detection between the two different phase detection schemes. First the local excitation phase is detected on the photomultiplier tube (PMT), when AOM 2 passes all OPO-signal power, and second, the heterodyne phase is detected on the photodiode, when AOM 2 passes only a few nW. By switching and sample-hold electronics the overload during the excitation phase detection time on the photodiode is removed. The detection of the local excitation phase is done by a second lock-in amplifier at the same $100 \mathrm{kHz}$ modulation. Both lock-in amplifiers are set to $1 \mathrm{~ms}$ integration time. Shorter pixel dwell times can be obtained by higher external modulation frequencies and shorter integration times, when different lock-in amplifiers are used.

Figure 3 shows fixed HeLa cells in water that contain lipid droplets of various sizes. CARS images of $50 \times$ $50 \mu \mathrm{m}, 262 \times 262$ pixels with a 1.2 NA water objective, are acquired of these cells at $2845 \mathrm{~cm}^{-1}$. The detected heterodyne phase on the photodiode, Fig. 3(a), shows that the phase over the field of view is not constant, but distorted and noisy. At the location of the lipids in the cell we can see phase steps. These phase steps are mostly due to the difference in phase between resonant and nonresonant signals. Figure 3(b) shows the local excitation phase detected on the PMT which is also distorted and noisy. Again phase steps are observed at the location of the lipid droplets, but these phase steps are caused by the difference in refractive index between lipid and the water in the cell. The amplitude image of the CARS signal is shown in Fig. 3(c). There is contrast between the cells and the surrounding water, and the internal structure within the cells can be observed despite the lack of resonant material there. Resonant lipid droplets can easily be seen, as the amplitude of many droplets exceeds the nonresonant signal. Interferences on the edges of the droplets yield a "dark" ring around the droplets, enhancing the contrast. Note that there are also droplets represented by dark spots in the amplitude image. Subtraction of the local excitation phase

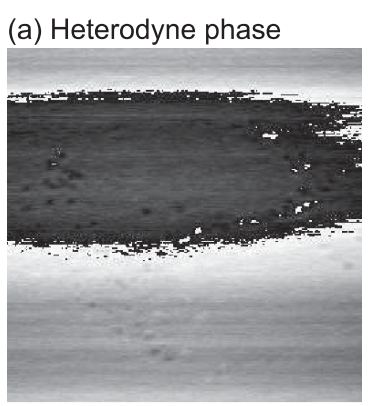

(c) Amplitude

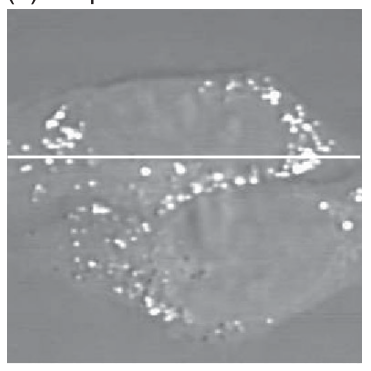

(e) Background free

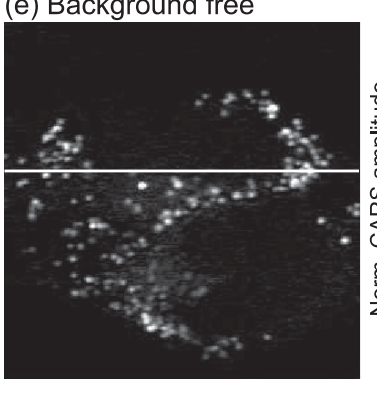

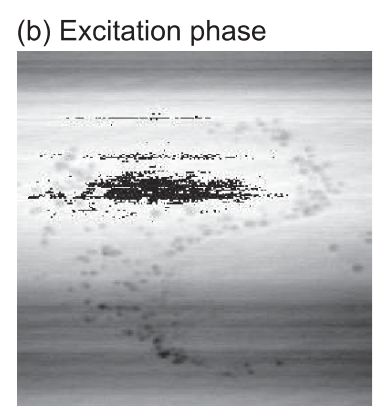

(d) Vibrational phase

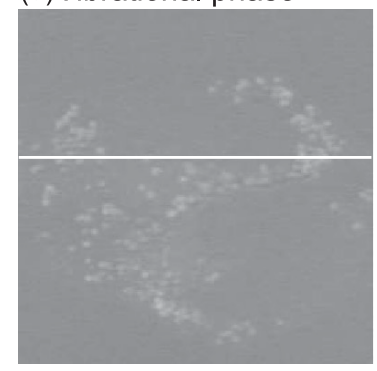

(f) Line cross sections

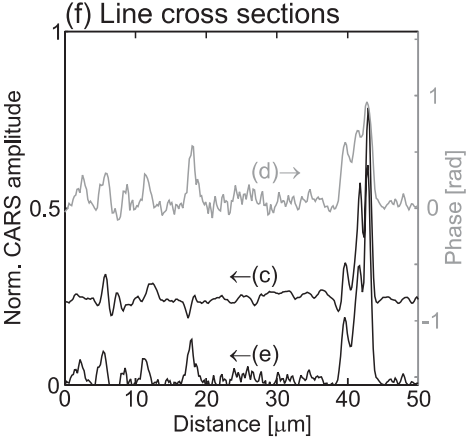

FIG. 3. Fixed HeLa cells in water with various sizes of lipid droplets imaged at $2845 \mathrm{~cm}^{-1}$. (a and b) are, respectively, a single oscillator and local excitation phase image. (c and d) are, respectively, a 5 times averaged CARS amplitude and vibrational phase image (subtraction of the local excitation phase with the oscillator phase). (e) Background free amplitude. (f) Line cross sections of images (c, d, and e). The scaling of the phase images $(\mathrm{a}, \mathrm{b}$, and $\mathrm{d})$ are the same. The scaling of the amplitude images (c and e) are also the same.

from the oscillator phase can be seen in Fig. 3(d). The distortion and noise are canceled, allowing for averaging of this vibrational phase image to increase the precision on the phase value. In this image the phase of the pure nonresonant water signal outside the cell is used to pinpoint phase zero and subtract a residual phase difference caused by the AOM switching between different levels. Since there is a strong nonresonant background, the phase step at the lipids does not reach $\pi / 2$ [see Fig. 3(f), line cross section (d)]. Equation (3) shows that taking the sine of the vibrational phase image and multiplying it with the amplitude image removes the nonresonant signal, which is done in Fig. 3(e). Comparing the amplitude image and the background free image, it can be seen that the internal 
structure of the cell and the edge of the cell have disappeared and only the resonant lipids can be observed. This shows that the cell structures in these amplitude images are nonresonant contributions. This profile should not be confused with the distribution of resonant material at the cell boundary. The line cross section [see Fig. 3(f)] shows that the nonresonant amplitude is lowered from 0.25 to less than 0.02. The three lipid droplets in the line cross section around $42 \mu \mathrm{m}$ show only a small drop in amplitude due to the $\sim \pi / 4$ phase step at these locations. The region between 0 and $15 \mu \mathrm{m}$ shows lipid droplets that are faint compared to the nonresonant signal, but stand out when the nonresonant signal is removed. The dispersive feature at $18 \mu \mathrm{m}$ is accompanied by a phase step, resulting in a recovered clean amplitude in the background free image.

In conclusion we demonstrated a cascaded phasepreserving chain to obtain the phase of oscillating molecules with respect to the excitation field for background free imaging. Two phase detection schemes are alternated on a point-by-point base, so that the acquired phase is corrected for refractive index changes in the sample, phase curvature over the field of view, and interferometric instabilities. The phase detection improves the signal-tobackground by an order of magnitude allowing for imaging of concentrations 10 times lower than previously attainable.

This research is supported by NanoNed, a nanotechnology programme of the Dutch Ministry of Economic Affairs and partly financed by the Stichting voor Fundamenteel Onderzoek der Materie (FOM), which is financially supported by the Nederlandse Organisatie voor Wetenschappelijk Onderzoek (NWO). We also acknowledge Coherent Inc. for the use of the Paladin laser and APE Berlin for the collaboration and use of a Levante Emerald OPO. Futhermore we would like to thank V. V. Pully for preparation of the sample. *h.1.offerhaus@tnw.utwente.nl

[1] T. Hellerer, C. Axaeng, C. Brackmann, P. Hillertz, M. Pilon, and A. Enejder, Proc. Natl. Acad. Sci. U.S.A. 104, 14658 (2007).

[2] X. Nan, E. Potma, and X. Xie, Biophys. J. 91, 728 (2006).

[3] M. Muller and A. Zumbusch, Chem. Phys. Chem. 8, 2156 (2007).

[4] A. Volkmer, J. X. Cheng, and X. S. Xie, Phys. Rev. Lett. 87, 023901 (2001).

[5] S. Akhmanov, A. Bunkin, S. Ivanov, and N. Koroteev, Sov. Phys. JETP 47, 667 (1978)

[6] A. Chikishev, G. Lucassen, N. Koroteev, C. Otto, and J. Greve, Biophys. J. 63, 976 (1992).

[7] J. Cheng, L. Book, and X. Xie, Opt. Lett. 26, 1341 (2001).

[8] A. Volkmer, L. Book, and X. Xie, Appl. Phys. Lett. 80, 1505 (2002).

[9] C. W. Freudiger, W. Min, B. G. Saar, S. Lu, G. R. Holtom, C. He, J. C. Tsai, J. X. Kang, and X. S. Xie, Science 322, 1857 (2008)

[10] P. Nandakumar, A. Kovalev, and A. Volkmer, New J. Phys. 11, 033026 (2009).

[11] O. Burkacky, A. Zumbusch, C. Brackmann, and A. Enejder, Opt. Lett. 31, 3656 (2006).

[12] F. Ganikhanov, C. Evans, B. Saar, and X. Xie, Opt. Lett. 31, 1872 (2006).

[13] E. Potma, C. Evans, and X. Xie, Opt. Lett. 31, 241 (2006).

[14] F. Zernike, Physica 9, 686 (1942).

[15] E. Andresen, S. Keiding, and E. Potma, Opt. Express 14, 7246 (2006).

[16] M. Jurna, J. P. Korterik, C. Otto, and H. L. Offerhaus, Opt. Express 15, 15207 (2007).

[17] F. Lu, W. Zheng, and Z. Huang, Appl. Phys. Lett. 92, 123901 (2008).

[18] M. Jurna, J. P. Korterik, C. Otto, J.L. Herek, and H. L. Offerhaus, Opt. Express 16, 15863 (2008).

[19] M. Jurna, E. Buttner, J. Korterik, C. Otto, I. Rimke, and H. Offerhaus, Proc. SPIE Int. Soc. Opt. Eng. 6860, 68600R (2008). 